\title{
"No es solo pitar": diseño e implementación de un taller de formación para árbitros y jueces de deporte escolar
}

\author{
Marta Borrueco, Saül Alcaraz, Yago Ramis y Jaume Cruz \\ Universitat Autònoma de Barcelona, España
}

RESUMEN: El objetivo de este trabajo es explicar cómo a partir de una demanda realizada por la Unió de Consells Esportius Catalans (UCEC), el Grup d'Estudis en Psicologia de l'Esport i de l'Activitat Física (GEPE) diseñó e implementó un taller de formación dirigido a árbitros y jueces de deporte escolar. Para abordar esta demanda, se utilizó como guía el Modelo GEPE de Práctica Basada en la Evidencia, modelo de consultoría que integra los últimos avances científicos del ámbito en la práctica aplicada. Para dar respuesta a la demanda planteada por la UCEC se siguieron las cinco fases del modelo: (1) Actualización documental, (2) Análisis de necesidades, (3) Intervención, (4) Evaluación y, (5) Seguimiento. Un total de 40 árbitros y jueces de deporte escolar participaron en los cinco talleres de formación realizados, mostrándose satisfechos con el mismo. Este trabajo puede servir de ejemplo para aquellos profesionales que quieran basar su práctica en la evidencia científica para así dotarla de rigurosidad, teniendo en cuenta además las necesidades de las personas implicadas en el proceso.

PALABRAS CLAVES: práctica basada en la evidencia, árbitros y jueces, análisis de necesidades, taller de formación.

\section{"It's not just taking decisions": design and implementation of a training workshop for school sports} officials and judges

ABSTRACT: The purpose of this paper is to describe how the GEPE (Research Group in Sport and Exercise Psychology), in response to a demand from the UCEC (Group of Sport Councils of Catalunya), designed and implemented a workshop to train officials and judges in Catalan school sports. The GEPE Model of Evidence-Based Practice, integrating the most recent scientific advances in the applied practice, was used to address this demand. This consultancy model consists of five steps: (1) Literature update, (2) Needs analysis, (3) Intervention, (4) Assessment and, (5) Monitoring. A total of 40 officials and judges participated in the five conducted workshops and were very satisfied with them. This work may serve as a guide for professionals who want to base their practice on scientific evidence to make it more rigorous while taking into account the needs of the people involved in the whole process.

KEYWORDS: evidence-based practice, officials and judges, needs analysis, training workshop.

\section{"Não se trata apenas de apitar": desenvolvimento e implementação de um workshop de formação para árbitros e juízes de desportos escolares}

RESUMO: O objetivo deste trabalho é explicar como a partir de um pedido feito pela Unió de Consells Esportius Catalans (UCEC), o Grup d'Estudis en Psicologia de l'Esport i de l'Activitat Física (GEPE) desenvolveu e implementou um workshop de formação destinado a árbitros e juízes de desportos escolares. Para responder a esse pedido, foi usado como guia o Modelo GEPE de Prática Baseada em Provas, um modelo de consultoria que integra os mais recentes avanços científicos no âmbito da prática aplicada. Para dar resposta ao pedido

\footnotetext{
Financiación: Este trabajo ha sido posible gracias al convenio de colaboración entre la Universitat Autònoma de Barcelona y la Unió de Consells Esportius de Catalunya (2017).

Marta Borrueco, Saül Alcaraz, Yago Ramis y Jaume Cruz son psicóloga y psicólogo en el Grup d'Estudis en Psicologia de l'Esport i de l'Activitat Física (GEPE) de la Universitat Autònoma de Barcelona.

La correspondencia sobre este artículo debe enviarse a la primera autora al Departament de Psicologia Bàsica, Evolutiva i de l'Educació, Facultat de Psicologia Edifici B, Universitat Autònoma de Barcelona, 08193, Bellaterra (Cerdanyola del Vallès), Barcelona (Spain). E-mail:
} 
feito pela UCEC, foram seguidas as cinco fases do modelo: (1) atualização documental, (2) análise de necessidades, (3) intervenção, (4) avaliação e (5) acompanhamento. Um total de 40 árbitros e juízes de desportos escolares participaram nos cinco workshops de formação realizados, mostrando-se satisfeitos com os mesmos. Este trabalho pode servir de exemplo para os profissionais que queiram sustentar a sua prática em provas científicas, a fim de conferir rigor à mesma, tendo também em consideração as necessidades das pessoas envolvidas no processo.

PALAVRAS-CHAVE: prática baseada em provas, árbitros e juízes, análise de necessidades, workshop de formação.

Artículo recibido: 04/08/2019 | Artículo aceptado: 02/11/2019

La práctica de deporte se relaciona con beneficios a largo plazo a nivel de rendimiento (i.e., aprendizaje motor), de participación (i.e., estilo de vida activo) y de desarrollo personal (i.e., psicosocial y emocional), constituyéndose así como un entorno adecuado para el desarrollo de los jóvenes (e.g., Côté y Hancock, 2014; Côté, Strachan y Fraser-Thomas, 2007; Harwood y Johnston, 2016). Además, el contexto deportivo supone una gran oportunidad para el fomento de aspectos relacionados con el desarrollo moral (e.g., Cruz, Ramis y Torregrosa, 2016; Hodge y Gucciardi, 2015; Kavussanu, Roberts y Ntoumanis, 2002). Un escenario idóneo para promover un deporte centrado en el desarrollo integral de los deportistas es el deporte escolar ya que, por definición, pretende "impulsar un modelo deportivo que incluya la vertiente educativa, recreativa y competitiva" (DOGC núm. 3546, 2002, p.155).

La Unió de Consells Esportius Catalans (Unión de Consejos Deportivos Catalanes; UCEC) es la organización responsable de coordinar las competiciones escolares de Catalunya. A diferencia del deporte federado, regulado por las federaciones y de carácter competitivo, el deporte escolar se define como un deporte educativo. El deporte escolar, sin dejar de lado la competición, tiene como fin último la diversión de los niños y niñas que lo practican, así como la promoción de determinados valores asociados con el deporte (e.g., equidad, esfuerzo, empatía). Nuestro grupo de investigación, el Grup d'Estudis en Psicologia de l'Esport i de l'Activitat Física (Grupo de Estudios en Psicología del Deporte y de la Actividad Física; GEPE) recibió la demanda por parte de la UCEC de desarrollar una propuesta formativa dirigida a árbitros y jueces escolares de Catalunya, Ilamados Tutors/es de Joc (Tutores/ as de Juego; TJ) para resaltar su papel pedagógico, centrada tanto en la formación en valores como en aspectos psicológicos, con la intención de unificar las distintas formaciones que ofrecían, hasta ese momento, los Consells Esportius (Consejos Deportivos; CE) de cada comarca.

Para dar respuesta a la demanda propuesta por la UCEC, se utilizó como guía el Modelo GEPE de Práctica Basada en la Evidencia (Modelo GEPE de PBE: Ramis, Torregrossa, Pa-
Ilarès, Viladrich y Cruz, 2019, ver Figura 1) estructurado en cinco fases: (1) Actualización documental a partir de la revisión de trabajos y publicaciones sobre la figura del árbitro/ juez (2) Análisis de necesidades mediante reuniones con la organización deportiva y grupos focales con los usuarios finales, (3) Intervención en forma de taller educativo , (4) Evaluación de la satisfacción de los participantes en el taller y, (5) Seguimiento de la implementación de la propuesta de intervención.

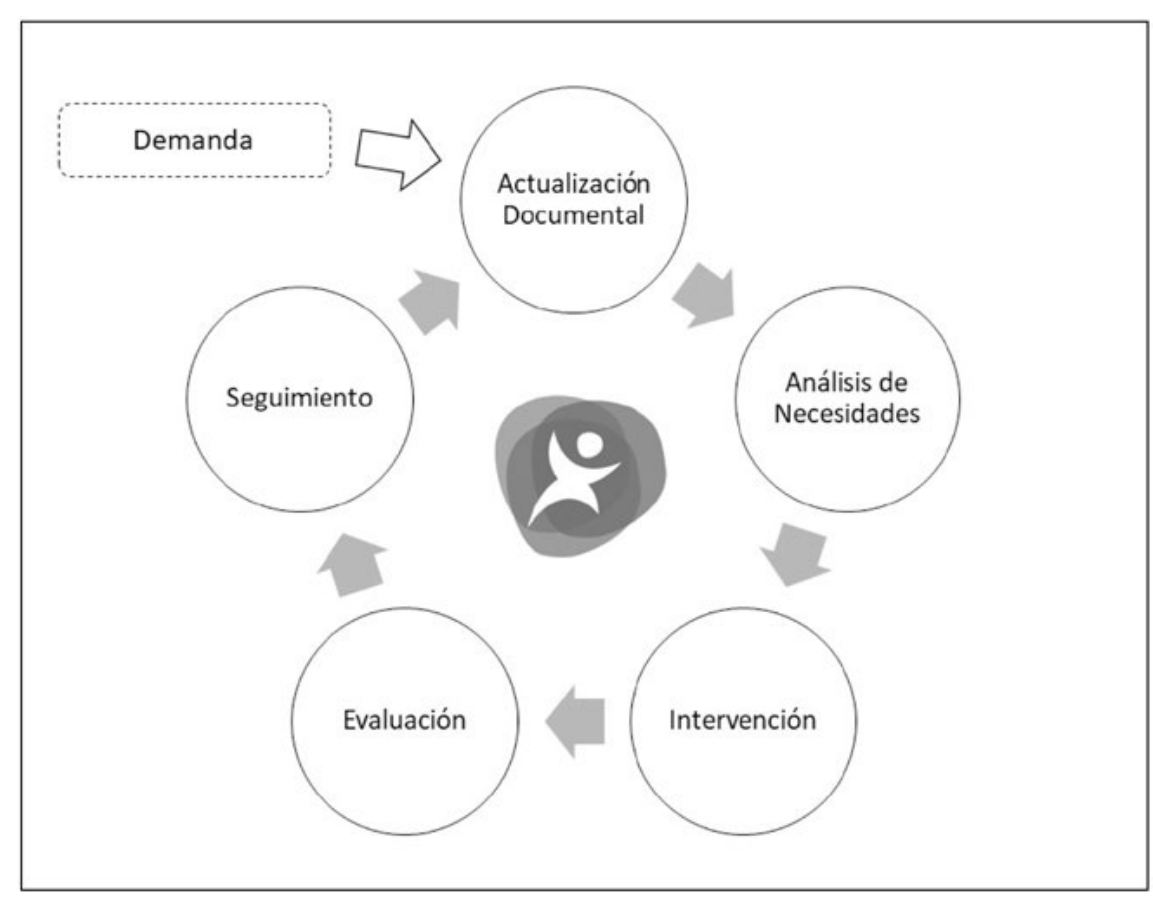

\section{Figura 1. Modelo GEPE de Práctica Basada en la Evidencia}

Nota. Reproducida con el permiso de los autores/as de "El Modelo GEPE de práctica basada en la evidencia: Integrando evidencia científica y práctica aplicada" de Ramis et al., 2019 en la Revista de Psicología Aplicada al Deporte y al Ejercicio Físico.

\section{Experiencia profesional}

En una primera reunión entre la UCEC y el grupo de investigación se acordó en qué se traducía concretamente la demanda (i.e., taller de formación) y cómo se abordaría (i.e., Modelo GEPE de PBE, Ramis et al., 2019), así como las condiciones del convenio de colaboración entre la UCEC y el GEPE 
(e.g., presupuesto, personas implicadas). Desde un principio, se acordó dividir el proyecto en dos fases. En la primera fase, el GEPE realizó una formación para árbitros y jueces escolares expertos, que recibieron una serie de herramientas y contenidos para ejercer posteriormente como formadores del resto de árbitros y jueces escolares (i.e., segunda fase de la propuesta formativa). Por tanto, el trabajo del grupo de investigación se centró exclusivamente en la primera fase de formación, actuando como formador de formadores.

\section{Actualización documental}

Para este proyecto se tomaron como referencia trabajos enmarcados en la Teoría de la Autodeterminación (SDT; en inglés Self-Determination Theory; Ryan y Deci, 2017) para explicar el rol del árbitro/juez como agente social y transmisor de valores. Para trabajar las habilidades psicológicas se utilizó literatura actual sobre la tarea del árbitro/juez (e.g., Hancock, Dawson y Auger, 2015; Guillén y Feltz, 2011; Soriano, Ramis, Torregrossa y Cruz, 2018).

El rol del árbitro/juez como agente social y transmisor de valores. El árbitro o juez es un agente presente en el entorno del deportista, sobre todo durante la competición y, por tanto, puede influir en su experiencia deportiva. La SDT postula que los agentes sociales presentes en el entorno del deportista pueden potenciar que el deportista desarrolle una motivación intrínseca (i.e., autodeterminada) hacia su deporte mediante la satisfacción de tres necesidades psicológicas básicas (BPN; en inglés Basic Psychological Needs): (a) autonomía (i.e., sentir que tu opinión se tiene en cuenta; De Charms, 1968), (b) competencia (i.e., sentirte capaz de resolver tu tarea con eficacia; White, 1959) y, (c) relación (i.e., sentir que perteneces a un grupo; Baumeister y Leary, 1995). Además, investigaciones basadas en la satisfacción de BPN se relacionan con el desarrollo de comportamientos prosociales (Hodge y Gucciardi, 2015; Hodge y Lonsdale, 2011; Roth, Kanat-Maymon y Bibi, 2011); es decir, actuar acorde a los valores considerados como positivos en el deporte (e.g., empatía, cooperación, respeto; Kavussanu y Stanger, $2017 a, b)$ y el bienestar (e.g., Ryan y Deci, 2001). Finalmente, la motivación autodeterminada también se ha relacionado con el compromiso y el esfuerzo dirigido hacia una actividad, disminuyendo así la probabilidad de abandono de la misma (Beniscelli y Torregrossa 2010; Soriano, Ramis, Torregrossa y Cruz, 2019).

La tarea del árbitro/juez en el deporte. El árbitro/juez realiza una función esencial para el desarrollo óptimo de las competiciones deportivas (Soriano et al., 2018), siendo el responsable, entre otros aspectos, de evaluar si se están cumpliendo las normas y reglas del deporte interpretando correctamente las acciones que se llevan a cabo durante la competición y tratando de salvaguardar la integridad de los deportistas (Hancock, Rix-Lièvre y Côté, 2015). La complejidad de su tarea conlleva una gran probabilidad de cometer errores que se relaciona, a su vez, con una pérdida de confianza y niveles altos de ansiedad y estrés (Taylor y Daniel, 1988; Soriano et al., 2018), provocando en consecuencia un gran número de abandonos (Hancock et al., 2015; Titlebaum, Haberlin y Titlebaum, 2009). Para hacer frente a las demandas relacionadas con la tarea se utilizó el Modelo de Autoeficacia en Árbitros propuesto por Guillén y Feltz (2011). Basándose en la autoeficacia como predictor de la toma de decisiones rápidas y eficaces (Hepler y Chase, 2008), este modelo presenta cinco aspectos claves relacionados con el éxito en el arbitraje: (a) conocimiento sobre el deporte: conocer aspectos técnicos y tácticos del deporte en cuestión, así como el reglamento y la normativa; (b) habilidades estratégicas: saber dónde colocarse en el campo/pista, cómo moverse, realizar los gestos correctos y anticipar acciones es fundamental para interpretar el juego de forma correcta; (c) toma de decisiones: tomar decisiones con la mayor rapidez y eficacia posible; (d) habilidades psicológicas: focalizar la atención y trabajar la concentración, rendir bajo presión, manejar los errores y las malas decisiones y (e) habilidades de comunicación y de control del juego: conocer pautas para lograr una comunicación eficaz y saber controlar el ritmo y las situaciones de juego.

\section{Análisis de necesidades}

Para analizar las necesidades de formación vinculadas con la transmisión de valores y la tarea de arbitrar/juzgar se llevaron a cabo dos acciones: (a) una reunión con algunos responsables de los CE y, (b) dos grupos focales con varios árbitros y jueces de deporte escolar. En la reunión inicial se acordaron aspectos relacionados con la formación, por ejemplo se concretó cuál sería el diseño de la intervención, el número de sesiones, cuántos participantes asistirían aproximadamente en cada una de ellas y el calendario. Además, se pactó la realización de dos grupos focales para conocer las necesidades específicas de la población diana. Catorce árbitros y jueces de deporte escolar con distinto grado de experiencia (i.e., entre 1 y 20 años) contactados previamente por la UCEC participaron en los dos grupos focales, representando tanto a deportes individuales (e.g., patinaje artístico, gimnasia artística) como de equipo (e.g., baloncesto, futbol sala, balonmano). Se elaboró un guion de entrevista semi-estructurada donde se abordaron tres temas: (a) qué significaba 
para ellos ser árbitros/jueces de deporte escolar, (b) cómo creían que debían de actuar y (c) qué formación específica habían recibido y/o les gustaría recibir. Asimismo, antes de empezar los grupos focales, se les explicaron cuáles eran los objetivos del encuentro y se comunicó a las personas asistentes que su participación iba a ser grabada para su posterior análisis. Además, se les entregó el consentimiento informado para que lo firmaran y se les recordó que su participación era voluntaria y que podían abandonar el grupo en cualquier momento si así lo deseaban. Una vez finalizados ambos grupos focales, con una duración aproximada de 60 minutos cada uno, se procedió a la transcripción de las grabaciones.

Los resultados del análisis de los grupos focales mostraron que se percibían como agentes que debían evaluar y aplicar el reglamento actuando de forma pedagógica (i.e., explicando las decisiones y enseñando el reglamento a los/ las deportistas), trasmitiendo los valores positivos del deporte y buscando que los deportistas se divirtieran. Además, ser árbitro/juez escolar implicaba ser un ejemplo para los deportistas, tener empatía (e.g., conocer y a adaptarse a las necesidades de los deportistas), ser capaz de soportar la presión, mostrar seguridad y estar motivado/a intrínsecamente para desarrollar su tarea. A nivel de formación, destacaron la necesidad de tener una normativa y criterios más claros y objetivos y, además, recibir formación específica sobre cómo manejar las relaciones con otros agentes del entorno (i.e., entrenadores/as y familias).

\section{Intervención}

A partir de la información recogida en los grupos focales y la actualización documental, y en consonancia con las condiciones de la UCEC, se diseñó un taller de formación teórico-práctico dirigido a árbitros y jueces de deporte escolar estructurado en dos bloques: (a) el papel del árbitro y/o juez escolar en la transmisión de valores y (b) las habilidades psicológicas necesarias para desarrollar la tarea de árbitro/ juez. El taller tenía una duración de 4 horas y se impartió en 6 sedes de CE de Catalunya.

Desarrollo y contenido del taller. Cada taller seguía la misma estructura: (a) breve presentación sobre la figura del árbitro/juez escolar, su relación con el entorno (i.e., entrenadores/as y familias), sus funciones y características psicológicas, (b) la influencia del árbitro/juez en la experiencia deportiva del niño/a y el papel del deporte escolar como escenario idóneo para la transmisión de valores, (c) trabajo de habilidades psicológicas relacionadas con la tarea a desarroIlar, como por ejemplo la comunicación verbal y no verbal, el manejo del error y la gestión de conflictos, haciendo además especial énfasis en el control emocional y en la concentración debido a su destacada importancia en investigaciones científicas anteriores (Urra, Nuñez, Oses y Sarmiento, 2018; Vela y Arbinaga, 2018) y, (e) material para poder realizar una formación continuada (e.g., diario con actividades de seguimiento). Estos contenidos teóricos iban intercalándose con ejercicios donde se trabajaba cómo integrar los conocimientos que se iban adquiriendo en la práctica real. Finalmente, como se ha mencionado anteriormente, los participantes en el taller (i.e., primera fase de la intervención) serían los encargados de formar luego a árbitros y jueces escolares de su demarcación geográfica (i.e., segunda fase de la intervención), por lo que durante toda la sesión se iban dando pautas y recursos sobre cómo podían transmitir el contenido del taller a los destinatarios finales (i.e., otros árbitros/jueces escolares). Al finalizar el taller, se les administraba una encuesta para valorar su satisfacción, garantizando el anonimato en las respuestas e informando de la voluntariedad de realizarla. Los materiales (e.g., presentación en PowerPoint, dosier con los aspectos clave, y material adicional de profundización) se les envió al finalizar el proyecto, ya que se iban adaptando y modificando según los comentarios, sugerencias y dudas que iban surgiendo en cada taller, garantizando así la permeabilidad y la regulación de los materiales en función de la propia experiencia formativa.

\section{Evaluación}

Un total de 40 árbitros y jueces escolares (45\% mujeres) participaron en los talleres organizados en distintas sedes de CE del territorio de Cataluña (i.e., Mollerussa $=32.5 \%$; Barcelona $=12.5 \%$; Igualada $=32{ }^{\prime} 5 \%$; Manresa $=15 \%$ y Banyoles $=7.5 \%)$. El rango de edad de los participantes oscilaba entre los 18 y los 55 años $(M=30.3 ; D T=8.48)$. Para la evaluación del taller se diseñó una encuesta de satisfacción ad-hoc que se administró al finalizar cada taller. La encuesta se estructuraba en (a) nueve ítems donde se pedía a los participantes que valoraran, utilizando una escala de tipo Likert ( $1=$ Nada a $5=$ Mucho), diversos aspectos sobre el taller (e.g., satisfacción, aplicabilidad de los contenidos), y (b) dos preguntas de respuesta abierta donde evaluaban cualitativamente los aspectos mejor y peor valorados del taller.

En general, los participantes se mostraron satisfechos con el taller $(M=4.6 ; D T=0.55)$. Además, valoraron como aplicables a su realidad los conocimientos adquiridos en él $(M=4.5 ; D T=0.72)$ y percibieron la dinámica del taller como idónea para compartir experiencias entre ellos $(M=4.4 ; D T$ $=0.84$ ). Este último aspecto es relevante ya que aquellos ár- 
bitros/as y jueces que tienen la posibilidad de comunicarse e intercambiar experiencias, muestran un mayor compromiso con el desempeño de su tarea (Soriano et al., 2019). En relación a la parte de respuesta abierta, los participantes valoraron positivamente la naturaleza teórico-práctica de la formación, la importancia y calidad del material utilizado, así como la estructura y contenidos del taller. Por el contrario, los asistentes percibieron que era necesario tener más tiempo de formación, profundizar más en el contenido explicado y, finalmente, ofrecer más ejemplos sobre situaciones reales del día a día de su deporte en concreto.

\section{Seguimiento}

Pese a no ser parte de la demanda inicial, durante la fase de seguimiento se quiso conocer si se llegó a implementar la segunda fase de la intervención, es decir, si las personas asistentes al taller llegaron a ser formadoras. Aproximadamente un año y medio después de finalizar la primera fase de la intervención (i.e., talleres de formación) se contactó con dos participantes que habían asistido a la formación. En primer lugar, se pretendía conocer si habían llegado a actuar de formadores y, en caso de ser así, se evaluó la fidelidad (Durlak, 2010) de la implementación de los talleres según (a) el nivel de correspondencia entre la versión original del taller y la que implementaron (i.e., adherencia) y (b) la cantidad de información de la intervención que se implementó (i.e., dosis).

De los dos participantes, una contestó que durante el tiempo que estuvo en la entidad (i.e., hasta medio año después de la formación) no se llegó a poner en marcha la segunda fase de la intervención. En cambio, el segundo sí que llegó a actuar como formador de árbitros y jueces escolares. Realizó un taller de dos horas de duración un año después de que finalizara la primera fase, y utilizó todo el material (i.e., conceptos teóricos y actividades prácticas) que se le proporcionó manteniendo la estructura original, profundizando en aspectos que consideró novedosos e interesantes para los participantes (i.e., necesidades psicológicas básicas y auto-habla). Además, también valoró positivamente la utilidad del material teórico y actividades de formación continuada, ya que le ayudaron en su preparación para la implementación del taller.

\section{Discusión}

Este trabajo presenta un ejemplo de cómo el GEPE desarrolla actualmente su servicio de consultoría. Concreta- mente en esta experiencia profesional se explica cómo se abordó la recepción de la demanda de diseñar e implementar un taller de formación dirigido a árbitros y jueces de deporte escolar. En este caso, la demanda llegó a través de una organización deportiva (i.e., UCEC) y formaba parte de una propuesta de implementación global donde los árbitros/jueces que recibieron la formación en primera instancia, serían los formadores en la segunda fase de la intervención. Para abordar esta demanda, el grupo de investigación utilizó el Modelo GEPE en PBE (Ramis et al., 2019) con el fin de ofrecer una propuesta de práctica profesional aplicada basada en las últimas actualizaciones del ámbito.

La concepción de "formación de formadores" posibilita un aumento en el número de destinatarios finales beneficiados por la formación. En contraposición, que el grupo de investigación sea responsable solamente de una fase de la intervención dificulta la evaluación del impacto global de ésta. Se debe tener en cuenta que, en nuestra realidad de práctica profesional, como en cualquier modelo de consultoría, quién marca las áreas de actuación es el cliente (e.g., organización, club deportivo, federación), limitando y orientando la intervención hacia aquellas áreas que consideren más adecuadas y/o necesarias. Asimismo, este modelo de consultoría tiene la premisa de dotar de autonomía a la organización demandante con el fin de lograr gestionarse de forma autosuficiente (Ramis et al., 2019). Es por ello que la tarea del GEPE se centraba exclusivamente en desarrollar la primera fase de la intervención (i.e., taller de formación) mientras que la organización deportiva (i.e., UCEC) era la responsable de coordinar la segunda fase. La única información disponible sobre el desarrollo de la segunda fase se deriva de la información proporcionada por las dos personas contactadas durante la fase de seguimiento, siendo ésta insuficiente para obtener conclusiones concluyentes sobre si se llegó a implementar o no y de qué manera la segunda fase de la intervención en todos los CE de Catalunya.

Si bien es cierto que durante la fase de evaluación de la intervención se encontró que varios asistentes a los talleres valoraron que se explicaba demasiado contenido para el tiempo que duraba el taller (i.e., 4 horas) y sin profundizar lo suficiente en ellos, la estructura y contenido de la formación respondía a las necesidades de la población diana derivadas de los grupos focales. Durante la primera fase de la intervención se realizó un taller genérico para todos los árbitros y jueces escolares, es decir, no se tuvo en cuenta la idiosincrasia de cada modalidad deportiva. Aun así, se animó a los participantes a que, cuando actuaran como formadores, adaptaran el contenido a su deporte ya que hay aspectos que influyen más o menos según el tipo de deporte (e.g., ansiedad competitiva; Pons, Ramis, Garcia-Mas, López 
de la Llave, Pérez-Llantada, 2016; Ramis, Torregrossa y Cruz, 2013). Para solventar estas situaciones se ofrecieron materiales adicionales (e.g., material complementario, actividades extra, diario de seguimiento) orientados a la formación continuada, así como recursos para facilitar la aplicación de los contenidos del taller.

\section{Aplicaciones prácticas}

Es importante destacar que más allá de ofrecer un ejemplo de respuesta ante una demanda de intervención por parte de una organización deportiva, este trabajo también tiene el objetivo de defender que una práctica aplicada basada en la evidencia científica es posible y necesaria para dotar a nuestra profesión de rigurosidad conceptual y metodológica. Asimismo, también describe un ejemplo de cómo un modelo se puede utilizar de guía para orientar la práctica profesional, adaptando la selección de técnicas de análisis de necesidades (e.g., grupos focales), la propuesta de intervención (e.g., taller de formación), entre otras, a los objetivos de cada proyecto. Finalmente, y haciendo referencia al carácter recursivo del Modelo GEPE, este artículo pretende compartir y divulgar resultados que puedan servir como información actualizada (i.e., fase de actualización documental) para otros profesionales del ámbito en el momento de desarrollar sus propias intervenciones.

\section{Referencias}

Baumeister, R. F. y Leary, M. R. (1995). The need to belong: desire for interpersonal attachments as a fundamental human motivation. Psychological Bulletin, 177, 497-529. https://doi. org/10.1037/0033-2909.117.3.497

Beniscelli, B. y Torregrosa, M. (2010). Componentes del Esfuerzo Percibido en Fútbol de Iniciación. Cuadernos de Psicología del Deporte, 10(1), 7-21

Côté, J. y Hancock, D. J. (2014). Evidence-based policies for youth sport programmes. International Journal of Sport Policy and Politics, 8(1), 51-65. https://doi.org/10.1080/19406940.2014.9 19338

Côté, J., Strachan, L. y Fraser-Thomas, J. (2007). Participation, personal development, and performance through youth sport. En N. L. Holt (Ed.), Positive youth development through sport. (pp. 34-45). Londres, Reino Unido: Routledge.

Cruz, J., Ramis, Y. y Torregrosa, M. (2016). Barcelona's Campaign to Promote Parents' Sportspersonship in Youth Sports. En J. G. Cremades, y L. Tashman, (Eds.), Global Practices and Training in Applied Sport, Exercise, and Performance Psychology: A Case Study Approach (pp. 50-57). Nueva York, NY: Routledge.

DeCharms, R. (1968). Personal causation. The Internal Affective Determinants of Behavior. Nueva York, NY: Routledge.
Diari Oficial de la Generalitat de Catalunya (DOGC), núm. 3546 (2002). Recuperado de https://dogc.gencat.cat/es/pdogc_canals_interns/pdogc_sumari_del_dogc/?anexos $=1 \&$ selectedYear $=2002 \&$ selected Month $=0$ \&numDOGC $=3546 \&$ language $=$ es_ES\#

Durlak, J. A. (2010). The importance of doing well in whatever you do: A commentary on the special section "Implementation Research in Early Childhood Education." Early Childhood Research Quarterly, 25, 348-357. https://doi.org/10.1016/j. ecresa.2010.03.003

Guillén, F.y Feltz, D. L. (2011). A conceptual model of referee efficacy. Frontiers in Psychology, 2(25), 1-5. https://doi.org/10.3389/ fpsyg.2017.00025

Hancock, D. J., Dawson, D. J. y Auger, D. (2015). Why Ref? Understanding sport officials' motivations to begin, continue, and quit. Movement \& Sport Sciences-Science \& Motricité, 87, 31-39. https://doi.org/10.3917/sm.087.0031

Hancock, D. J., Rix-lièvre, G.y Côté, J. (2015). Citation network analysis of research on sport officials: a lack of interconnectivity. International Review of Sport and Exercise Psychology, 9858(Abril), 37-41. https://doi.org/10.1080/1750984X.2015.1022202

Harwood, C. y Johnston, J. (2016). Positive Youth Development and Talent Development: Is there a best of both worlds? En N. L. Holt (Ed.), Positive youth development through sport (pp. 113-125). Nueva York, NY: Routledge. https://doi. org/10.4324/9781315709499-10

Hepler, T. J. y Chase, M. A. (2008) Relationship between decision-making, task self-efficacy, and the performance of a sport skill. Journal of Sport Sciences, 26(6), 603-610. https://doi. org/10.1080/02640410701654280

Hodge, K. y Gucciardi, D. F. (2015). Antisocial and prosocial behavior in sport: the role of motivational climate, basic psychological needs, and moral disengagement. Journal of Sport and Exercise Psychology, 37, 257-273. https://doi.org/10.1123/ jsep. 2014-0225

Hodge, K. y Lonsdale, C. (2011). Prosocial and antisocial behavior in sport: the role of coaching style, autonomous vs. controlled motivation, and moral disengagement. Journal of Sport and Exercise Psychology, 33, 527-547. https://doi.org/10.1123/ isep.33.4.527

Kavussanu, M., Roberts, G. C. y Ntoumanis, N. (2002). Contextual influences on moral functioning of college basketball players. The Sport Psychologist, 16, 347-367. https://doi.org/10.1123/ tsp. 16.4.347

Kavussanu, M. y Stanger, N. (2017a). Moral behavior in sport. Current Opinion in Psychology, 16, 185-192. https://doi.org/10.1016/i.copsyc.2017.05.010

Kavussanu, M. y Stanger, N. (2017b). Prosocial and antisocial behaviors in sport organizations. En C. R. D. Wagstaff (Ed.), The organizational psychology of sport: Key issues and practical applications (pp. 176-192). Londres, Reino Unido: Routledge.

Pons, J., Ramis, Y., Garcia-Mas, A., López de la Llave, A. y Pérez-Llantada, M. C. (2016). Percepción de la Ansiedad Competitiva en relación al Nivel de Cooperación y Compromiso Deportivo en Jugadores de Baloncesto de Formación. Cuadernos de Psicología del Deporte, 16(3), 45-54.

Ramis, Y., Torregrossa, M. y Cruz, J. (2013). Revisitando a Simon \& Martens: la ansiedad competitiva en deportes de iniciación. Revista de Psicología del Deporte, 22(1), 77-83.

Revista de Psicología Aplicada al Deporte y al Ejercicio Físico (2019), 4, e12, 1-7 
Ramis, Y., Torregrossa, M., Pallarés, S., Viladrich, C. y Cruz, J. (2019). El modelo GEPE de práctica basada en la evidencia: Integrando la evidencia científica en la práctica aplicada. Revista de Psicología Aplicada al Deporte y al Ejercicio Físico, 4, e13 https://doi.org/10.5093/rpadef2019a12

Roth, G., Kanat-Maymon, Y. y Bibi, U. (2011). Prevention of school bullying: The important role of autonomy-supportive teaching and internalization of pro-social values. British Journal of Educational Psychology, 81, 654-666. https://doi.org/10.1348/20448279.002003

Ryan, R. M. y Deci, E. L. (2001). On happiness and human potentials: A review of research on hedonic and eudaimonic well-being. En S. Fiske (Ed.), Annual Review of Psychology (Vol. 52, pp. 141-166). Palo Alto, CA: Annual Reviews, Inc. https://doi. org/10.1146/annurev.psych.52.1.141

Ryan, R. M. y Deci, E. L. (2017). Self-determination theory: Basic psychological needs in motivation, development, and wellness. Nueva York, NY: Guilford Publications.

Soriano, G., Ramis, Y., Torregrossa. M. y Cruz, J. (2018). Fuentes de estrés dentro y fuera del partido en árbitros de fútbol. Apunts. Educació Física i Esports, 2(132), 22-31. https://doi. org/10.5672/apunts.2014-0983.es.(2018/2).132.02

Soriano, G., Ramis, Y., Torregrossa, M. y Cruz, J. (2019). El Lado Positivo del Arbitraje: Percepción de Apoyo Organizacional,
Motivación y Compromiso. Revista de Psicología del Deporte, 28(1), 41-50.

Taylor, A. H. y Daniel, J. V. (1988). Sources of stress in soccer officiating: an empirical study. En T. Reilly, A. Lees, K. Davis y W. J. Murphy (Eds.), Science and football: Proceedings of the First World Congress of Science and Football (pp. 538-544). Londres, Reino Unido: E. Y F. N. Spon.

Titlebaum, P. J., Haberlin, N. y Titlebaum, G. (2009). Recruitment and retention of sports officials. Recreational Sports Journal, 33(2), 102-108. https://doi.org/10.1123/rsj.33.2.102

Urra, B., Nuñez, C., Oses, J. y Sarmiento, G. (2018). Variables psicológicas influyentes en el desempeño de árbitros FIFA: un estudio cualitativo. Revista de Psicología Aplicada al Deporte y al Ejercicio Físico, 3, e15. https://doi.org/10.5093/rpadef2018a13

Vela, D. y Arbinaga, F. (2018). Aplicación del listado de situaciones estresantes para el arbitraje (LISEA) en árbitros de fútbol. Revista Iberoamericana de Psicología del Ejercicio y el Deporte, 13(2), 281-287.

White. R. W. (1959). Motivation reconsidered: The concept of competence. Psychological Review, 66, 297-333. https://doi. org/10.1037/h0040934 